\title{
Estimates of initial coefficients for certain subclasses of bi-univalent functions involving quasi-subordination
}

\author{
Obaid Algahtani \\ Department of Mathematics, College of Science, King Saud University, P. O. Box 231428, Riyadh 11321, Saudi Arabia. \\ Communicated by A. Atangana
}

\begin{abstract}
The object of the present paper is to introduce and investigate new subclasses of the function class $\Sigma$ of bi-univalent functions defined in the open unit disk $\mathcal{U}$, involving quasi subordination. The coefficients estimate $\left|a_{2}\right|$ and $\left|a_{3}\right|$ for functions in these new subclasses are also obtained. (C)2017 All rights reserved.
\end{abstract}

Keywords: Univalent functions, bi-univalent functions, quasi-subordination, subordination.

2010 MSC: 30C45, 30C50.

\section{Introduction}

Let $\mathcal{A}$ be the class of all functions of the form

$$
f(z)=z+\sum_{n=2}^{\infty} a_{n} z^{n},
$$

which are analytic in the open unit disk $\mathcal{U}=\{z \in \mathbb{C} ;|z|<1\}$.

Suppose $\mathcal{S}$ denotes the class of all functions of $\mathcal{A}$ which satisfy normalized condition $f(0)=0$ and $f^{\prime}(0)=1$ which are univalent in $\mathcal{U}$.

Since univalent functions are one-to-one, they are invertible and the inverse functions need not be defined on the entire unit disk $\mathcal{U}$. In fact, the Koebe-one quarter theorem [1] ensures that the image of $\mathcal{U}$ under every univalent function $f \in \mathcal{A}$ contains a disk of radius $1 / 4$. Thus, every univalent function $f$ has an inverse $f^{-1}$ satisfying $f^{-1}(f(z))=z,(z \in \mathcal{U})$, and

$$
f\left(f^{-1}(w)\right)=w \quad\left(|w|<r_{0}(f), r_{0}(f) \geqslant 1 / 4\right) .
$$

In fact, the inverse function $\mathrm{f}^{-1}$ is given by

$$
f^{-1}(w)=w-a_{2} w^{2}+\left(2 a_{2}^{2}-a_{3}\right) w^{3}-\left(5 a_{2}^{3}-5 a_{2} a_{3}+a_{4}\right) w^{4}+\cdots .
$$


A function $\mathrm{f} \in \mathcal{A}$ is said to be bi-univalent in $\mathcal{U}$ if both $\mathrm{f}$ and $\mathrm{f}^{-1}$ are univalent in $\mathcal{U}$. Let $\Sigma$ denote the class of bi-univalent functions defined in $\mathcal{U}$.

An analytic function $f(z)$ is subordinate to an analytic function $g(z)$ if there exists an analytic function $w(z)$ in $\mathcal{U}$ satisfying $w(0)=0$ and $|w(z)|<1(z \in \mathcal{U})$ satisfying $f(z)=g(w(z))$. We denote this subordination by, cf. [5, p. 226],

$$
\mathrm{f}(z) \prec \mathrm{g}(z) \quad(z \in \mathcal{U}) .
$$

Further, a function $f(z)$ is said to be quasi-subordinate to $g(z)$ in the open unit disk $\mathcal{U}$ if there exists an analytic function $\varphi(z)$ such that $f(z) / \varphi(z)$ is analytic in $\mathcal{U}$,

$$
\frac{f(z)}{\varphi(z)} \prec g(z) \quad(z \in \mathcal{U})
$$

and $|\varphi(z)| \leqslant 1(z \in \mathcal{U})$. We also denote this quasi-subordination by

$$
f(z) \prec_{\mathrm{q}} \mathrm{g}(z) \quad(z \in \mathcal{U})
$$

Note that the quasi-subordination (1.2) is equivalent to

$$
f(z)=\varphi(z) g(w(z)) \quad(z \in \mathcal{U}),
$$

where $|\varphi(z)| \leqslant 1(z \in \mathcal{U})$.

In the quasi-subordination (1.3), if $\varphi(z) \equiv 1$, then (1.3) becomes the subordination (1.3).

For analytic functions $f(z)$ and $g(z)$ in $\mathcal{U}$, we say $f(z)$ is majorized by $g(z)$ if there exists an analytic function $\varphi(z)$ in $\mathcal{U}$ satisfying $|\varphi(z)| \leqslant 1$ and $f(z)=\varphi(z) g(z)(z \in \mathcal{U})$. See [7, 11, 12] for works related to quasi-subordination.

Lewin [8] investigated the bi-univalent function class $\Sigma$ and showed that $\left|a_{2}\right|<1.51$. Subsequently, Brannan et al. [1] conjectured that $\left|a_{2}\right|<\sqrt{2}$. Netanyahu [10], on the other hand, showed that $\max _{\mathrm{f} \in \Sigma}\left|\mathrm{a}_{2}\right|=$ $\frac{4}{3}$. Brannan and Taha [2] obtained initial coefficient bounds for certain subclasses of bi-univalent functions, similar to the familiar subclasses of univalent functions consisting of strongly starlike, starlike and convex functions. Later, Srivastava et al. [13] introduced and investigated subclasses of bi-univalent functions and obtained bounds for the initial coefficients. Many researchers (see [3, 4, 6, 13, 14]) have recently introduced and investigated several interesting subclasses of the bi-univalent function class $\Sigma$ and they have found non-sharp estimates of initial coefficient bounds $\left|a_{2}\right|$ and $\left|a_{3}\right|$.

In the present paper, the coefficient bounds of $\left|a_{2}\right|$ and $\left|a_{3}\right|$ for certain classes involving the quasisubordination are obtained. The subclasses in this paper are motivated essentially by corresponding subclasses investigated in [7].

\section{Coefficient estimates}

Let us assume that there exists a function $\phi(z)$ analytic in the open unit disk $\mathcal{U}$ and $|\varphi(z)| \leqslant 1$ s.t.

$$
\phi(z)=A_{0}+A_{1} z+A_{2} z^{2}+\cdots \quad(|z|<1) .
$$

Since $\phi(z)$ is analytic and bounded in $\mathcal{U}$, we have [9, page 172]

$$
\left|A_{n}\right| \leqslant 1-\left|A_{0}\right|^{2} \leqslant 1 \quad(n>0) .
$$

Also, let $h(z)$ be an analytic function with positive real part in $\mathcal{U}$, satisfying $h(0)=1, h^{\prime}(0)>0$ and $h(\mathcal{U})$ is symmetric with respect to the real axis. Such a function has a Taylor series of the form

$$
\mathrm{h}(z)=1+\mathrm{B}_{1} z+\mathrm{B}_{2} z^{2}+\cdots, \mathrm{B}_{1}>0 .
$$

Suppose that $u(z)$ and $v(z)$ are analytic in $\mathcal{U}$ with $\mathfrak{u}(0)=v(0)=0,|\mathfrak{u}(z)|<1,|v(z)|<1$, and suppose that

$$
\mathrm{u}(z)=\mathrm{b}_{1} z+\sum_{\mathrm{n}=2}^{\infty} \mathrm{b}_{\mathrm{n}} z^{\mathrm{n}}, \quad v(z)=\mathrm{c}_{1} z+\sum_{\mathrm{n}=2}^{\infty} \mathrm{c}_{\mathrm{n}} z^{\mathrm{n}} \quad(|z|<1) .
$$


It is well-known that

$$
\left|b_{1}\right| \leqslant 1, \quad\left|b_{2}\right| \leqslant 1-\left|b_{1}\right|^{2}, \quad\left|c_{1}\right| \leqslant 1, \quad\left|c_{2}\right| \leqslant 1-\left|c_{1}\right|^{2} .
$$

By a simple calculation, we have

$$
h(u(z))=1+B_{1} b_{1} z+\left(B_{1} b_{2}+B_{2} b_{1}^{2}\right) z^{2}+\cdots,|z|<1,
$$

and

$$
h(v(w))=1+\mathrm{B}_{1} \mathrm{c}_{1} w+\left(\mathrm{B}_{1} \mathrm{c}_{2}+\mathrm{B}_{2} \mathrm{c}_{1}^{2}\right) w^{2}+\cdots,|w|<1 .
$$

We define a subclass of $\Sigma$ as follows:

Definition 2.1. A function $f \in \Sigma$ is said to be in the class $H_{q}(\Sigma, h)$ if and only if:

$$
f^{\prime}(z)-1 \prec_{\mathrm{q}} \mathrm{h}(z)-1, \quad \mathrm{~g}^{\prime}(w)-1 \prec_{\mathrm{q}} \mathrm{h}(w)-1,
$$

or

$$
f^{\prime}(z)-1=\phi(z)(h(u(z))-1), \quad g^{\prime}(w)-1=\phi(w)(h(v(w))-1),
$$

where $|\phi(z)| \leqslant 1(z \in \mathcal{U})$ and $g(w)=f^{-1}(w)$.

On taking $\phi(z) \equiv 1$ in Definition 2.1, we get the following subordination class:

$$
f^{\prime}(z) \prec h(z), \quad g^{\prime}(w) \prec h(w) .
$$

We name this class as $\mathrm{H}(\Sigma, \mathrm{h})$.

Now, we first derive following coefficient estimates for the subclass $H_{q}(\Sigma, h)$ :

Theorem 2.2. If $\mathrm{f}$ given by (1.1) is in the subclass $\mathrm{H}_{\mathrm{q}}^{\Sigma}(\mathrm{h})$, then

$$
\left|a_{2}\right| \leqslant \min \left\{\frac{B_{1}}{2}, \sqrt{\frac{B_{1}+\left|B_{2}\right|}{3}}\right\}, \quad\left|a_{3}\right| \leqslant \min \left\{\frac{B_{1}^{2}}{4}+\frac{2}{3} B_{1}, \frac{\left|B_{2}\right|}{3}+B_{1}\right\} .
$$

Proof. Let $f \in H_{q}^{\Sigma}(h)$ and $g=f^{-1}$. Then, there are analytic functions $u, v: \mathcal{U} \rightarrow \mathcal{U}$ given by (2.2) and a function $\phi(z)$ in $\mathcal{U}$ defined by (2.1) satisfying

$$
f^{\prime}(z)-1=\phi(z)\left(h(u(z)-1), \quad g^{\prime}(w)-1=\phi(w)(h(v(w)-1) .\right.
$$

Since

$$
f^{\prime}(z)=1+2 a_{2} z+3 a_{3} z^{2}+\cdots, \quad g^{\prime}(w)=1-2 a_{2} w+3\left(2 a_{2}^{2}-a_{3}\right) w^{2}+\cdots,
$$

and

$$
\phi(z)\left(h(u(z)-1)=A_{0} B_{1} b_{1} z+\left[A_{1} B_{1} b_{1}+A_{0}\left(B_{1} b_{2}+B_{2} b_{1}^{2}\right)\right] z^{2}+\cdots,\right.
$$

with

$$
\phi(w)\left(h(v(w)-1)=A_{0} B_{1} c_{1} w+\left[A_{1} B_{1} c_{1}+A_{0}\left(B_{1} c_{2}+B_{2} c_{1}^{2}\right)\right] w^{2}+\cdots\right.
$$

It follows that

$$
\begin{aligned}
2 a_{2} & =A_{0} B_{1} b_{1}, \\
3 a_{3} & =A_{1} B_{1} b_{1}+A_{0}\left(B_{1} b_{2}+B_{2} b_{1}^{2}\right), \\
-2 a_{2} & =A_{0} B_{1} c_{1}, \\
3\left(2 a_{2}^{2}-a_{3}\right) & =A_{1} B_{1} c_{1}+A_{0}\left(B_{1} c_{2}+B_{2} c_{1}^{2}\right) .
\end{aligned}
$$

From (2.6) and (2.8), we have

$$
\mathrm{b}_{1}=-\mathrm{c}_{1}
$$


Squaring and adding (2.6) and (2.8), and then using (2.10), we have

$$
4 \mathrm{a}_{2}^{2}=\mathrm{A}_{0}^{2} \mathrm{~B}_{1}^{2} \mathrm{c}_{1}^{2}
$$

Adding (2.7) and (2.9), we have

$$
6 a_{2}^{2}=A_{0}\left[B_{1}\left(b_{2}+c_{2}\right)+2 B_{2} c_{1}^{2}\right] .
$$

By using (2.2) and (2.3), we have from (2.11) and (2.12) that

$$
\left|a_{2}\right| \leqslant \frac{B_{1}}{2} \text { and }\left|a_{2}\right| \leqslant \sqrt{\frac{B_{1}+\left|B_{2}\right|}{3}}
$$

respectively. So we get the desired estimate on the coefficient $\left|a_{2}\right|$ as asserted in (2.1).

Next, in order to find the bound on the coefficient $\left|a_{3}\right|$, we subtract (2.9) from (2.7). Thus, we get

$$
6 a_{3}-6 a_{2}^{2}=A_{1} B_{1}\left(b_{1}-c_{1}\right)+A_{0}\left[B_{1}\left(b_{2}-c_{2}\right)+B_{2}\left(b_{1}^{2}-c_{1}^{2}\right)\right] .
$$

Therefore (2.10), (2.11), and (2.13) yield

$$
6 a_{3}=\frac{3}{2} A_{0}^{2} B_{1}^{2} c_{1}^{2}-2 c_{1} A_{1} B_{1}+A_{0} B_{1}\left(b_{2}-c_{2}\right) .
$$

Thus, we find by using (2.2) and (2.3) that

$$
\left|a_{3}\right| \leqslant \frac{1}{4} B_{1}^{2}+\frac{2}{3} B_{1} .
$$

Also from (2.10), (2.11), and (2.13), we have

$$
6 a_{3}=A_{0}\left[B_{1}\left(b_{2}+c_{2}\right)+2 B_{2} c_{1}^{2}\right]-2 c_{1} A_{1} B_{1}+A_{0} B_{1}\left(b_{2}-c_{2}\right) .
$$

Thus, we find by (2.2) and (2.3) that

$$
\left|a_{3}\right| \leqslant B_{1}+\frac{\left|B_{2}\right|}{3} .
$$

This evidently completes the proof of Theorem 2.2.

Theorem 2.3. If $\mathrm{f}$ given by (1.1) is in the subclass $\mathrm{H}(\Sigma, \mathrm{h})$ then

$$
\left|a_{2}\right| \leqslant \min \left\{\frac{B_{1}}{2}, \sqrt{\frac{B_{1}+\left|B_{2}\right|}{3}}\right\},
$$

and

$$
\left|a_{3}\right| \leqslant \min \left\{\frac{B_{1}^{2}}{4}+\frac{B_{1}}{6}, \frac{\left|B_{2}\right|+2 B_{1}}{3}\right\} .
$$

Proof. The result is obvious, by taking $\phi(z) \equiv 1(z \in \mathrm{U})$ and using the similar procedure as in Theorem 2.2.

Definition 2.4. A function $f \in \Sigma$ is said to be in the class $S_{q}^{*}(\Sigma, h)$ if and only if:

$$
\frac{z f^{\prime}(z)}{f(z)}-1 \prec_{q} h(z)-1, \quad \frac{w g^{\prime}(z)}{g(w)}-1 \prec_{q} h(w)-1,
$$

where $|\phi|(z) \leqslant 1(z \in \mathcal{U})$ and $g(w)=f^{-1}(w)$. 
On taking $\phi(z) \equiv 1$ in Definition 2.1, we get the following subordination class:

$$
\frac{z f^{\prime}(z)}{f(z)} \prec h(z), \quad \frac{w g^{\prime}(z)}{g(w)} \prec h(w) .
$$

We name this class as $S^{*}(\Sigma, h)$, where $g(w)=f^{-1}(w)$.

Theorem 2.5. If $\mathrm{f}$ given by (1.1) is in the subclass $\mathrm{S}_{\mathrm{q}}^{*}(\Sigma, \mathrm{h})$ then

$$
\left|a_{2}\right| \leqslant \min \left\{B_{1}, \sqrt{B_{1}+\left|B_{2}\right|}\right\}
$$

and

$$
\left|a_{3}\right| \leqslant \min \left\{B_{1}\left(1+B_{1}\right), 3 B_{1}\right\} .
$$

Proof. Let $f \in S_{q}^{*}(\Sigma, h)$ and $g=f^{-1}$. Then there are analytic functions $u, v: \mathcal{U} \rightarrow \mathcal{U}$ given by (2.2) and a function $\phi(z)$ in $\mathcal{U}$ defined by (2.1) satisfying

$$
\frac{z f^{\prime}(z)}{f(z)}-1=\phi(z)\left(h(u(z)-1), \quad \frac{w g^{\prime}(w)}{g(w)}-1=\phi(w)(h(v(w)-1) .\right.
$$

Since

$$
\frac{z f^{\prime}(z)}{f(z)}=1+a_{2} z+\left(2 a_{3}-a_{2}^{2}\right) z^{2}+\cdots, \quad \frac{w g^{\prime}(w)}{g(w)}=1-2 a_{2} w+\left(3 a_{2}^{2}-2 a_{3}\right) w^{2}+\cdots .
$$

By using (2.4), (2.5), (2.15), and (2.16), we have

$$
\begin{aligned}
a_{2} & =A_{0} B_{1} b_{1}, \\
2 a_{3}-a_{2}^{2} & =A_{1} B_{1} b_{1}+A_{0}\left(B_{1} b_{2}+B_{2} b_{1}^{2}\right), \\
-a_{2} & =A_{0} B_{1} c_{1} \\
3 a_{2}^{2}-2 a_{3} & =A_{1} B_{1} c_{1}+A_{0}\left(B_{1} c_{2}+B_{2} c_{1}^{2}\right) .
\end{aligned}
$$

From (2.17) and (2.19), we have

$$
\mathrm{b}_{1}=-\mathrm{c}_{1} .
$$

Squaring and adding (2.17) and (2.19), and then using (2.21), we have

$$
a_{2}^{2}=A_{0}^{2} B_{1}^{2} c_{1}^{2} .
$$

Adding (2.18) and (2.20), and then using (2.21), we have

$$
2 a_{2}^{2}=A_{0}\left[B_{1}\left(b_{2}+c_{2}\right)+2 B_{2} c_{1}^{2}\right] .
$$

By using (2.2) and (2.3), we have from (2.22) and (2.23) that

$$
\left|a_{2}\right| \leqslant B_{1} \text { and }\left|a_{2}\right| \leqslant \sqrt{B_{1}+\left|B_{2}\right|},
$$

respectively. So we get the desired estimate on the coefficient $\left|a_{2}\right|$ as asserted in (2.14).

Next, in order to find the bound on the coefficient $\left|a_{3}\right|$, we subtract (2.20) from (2.18). Thus, we get

$$
4 a_{3}-4 a_{2}^{2}=A_{1} B_{1}\left(b_{1}-c_{1}\right)+A_{0}\left[B_{1}\left(b_{2}-c_{2}\right)+B_{2}\left(b_{1}^{2}-c_{1}^{2}\right)\right] .
$$

Therefore, (2.21), (2.22), and (2.24) yield

$$
4 a_{3}=4 A_{0}^{2} B_{1}^{2} c_{1}^{2}-2 c_{1} A_{1} B_{1}+A_{0} B_{1}\left(b_{2}-c_{2}\right) .
$$


Hence, we find by using (2.2) and (2.3) that

$$
\left|a_{3}\right| \leqslant B_{1}\left(1+B_{1}\right) .
$$

Also from (2.21), (2.22), and (2.24), we have

$$
4 a_{3}=2 A_{0}\left[B_{1}\left(b_{2}+c_{2}\right)+2 B_{2} c_{1}^{2}\right]-2 c_{1} A_{1} B_{1}+A_{0} B_{1}\left(b_{2}-c_{2}\right) .
$$

Thus, we find by (2.2) and (2.3) that

$$
\left|a_{3}\right| \leqslant 3 B_{1} .
$$

This evidently completes the proof of Theorem 2.5.

Theorem 2.6. If $\mathrm{f}$ given by (1.1) is in the subclass $\mathrm{S}^{*}(\Sigma, \mathrm{h})$, then

$$
\left|a_{2}\right| \leqslant \min \left\{B_{1}, \sqrt{B_{1}+\left|B_{2}\right|}\right\}
$$

and

$$
\left|a_{3}\right| \leqslant \min \left\{B_{1}^{2}+\frac{B_{1}}{2}, \frac{3}{2} B_{1}+\left|B_{2}\right|\right\} .
$$

Proof. The result is obvious, by taking $\phi(z) \equiv 1(z \in \mathcal{U})$ and using the similar procedure as in Theorem 2.5.

Definition 2.7. A function $f \in \Sigma$ is said to be in the class $C_{q}(\Sigma, h)$ if and only if:

$$
\frac{z f^{\prime \prime}(z)}{f^{\prime}(z)} \prec_{q} h(z)-1, \quad \frac{w g^{\prime \prime}(z)}{g^{\prime}(w)} \prec_{q} h(w)-1,
$$

where $|\phi(z)| \leqslant 1(z \in \mathcal{U})$ and $g(w)=f^{-1}(w)$.

On taking $\phi(z) \equiv 1$ in Definition 2.1, we get the following subordination class:

$$
1+\frac{z f^{\prime \prime}(z)}{f^{\prime}(z)} \prec h(z), \quad 1+\frac{w g^{\prime \prime}(z)}{g^{\prime}(w)} \prec h(w) .
$$

We name this class as $C(\Sigma, h)$.

Theorem 2.8. If $\mathrm{f}$ given by $(1.1)$ is in the subclass $\mathrm{C}_{\mathrm{q}}(\Sigma, \mathrm{h})$, then

$$
\left|a_{2}\right| \leqslant \min \left\{\frac{B_{1}}{2}, \frac{\sqrt{B_{1}+\left|B_{2}\right|}}{2}\right\},
$$

and

$$
\left|\mathrm{a}_{3}\right| \leqslant \min \left\{\frac{1}{4} \mathrm{~B}_{1}^{2}+\frac{1}{3} \mathrm{~B}_{1}, \frac{5}{6} \mathrm{~B}_{1}+\frac{1}{2}\left|\mathrm{~B}_{2}\right|\right\} .
$$

Proof. Let $f \in K_{q}^{\Sigma}(h)$ and $g=f^{-1}$. Then, there are analytic functions $u, v: \mathcal{U} \rightarrow \mathcal{U}$ given by (2.2) and a function $\phi(z)$ in $\mathcal{U}$ defined by (2.1) satisfying

$$
\frac{z f^{\prime \prime}(z)}{f^{\prime}(z)}=\phi(z)\left(h(u(z)-1), \quad \frac{w g^{\prime \prime}(w)}{g^{\prime}(w)}=\phi(w)(h(v(w)-1)\right.
$$

Since

$$
\frac{z f^{\prime \prime}(z)}{f^{\prime}(z)}=2 a_{2} z+\left(6 a_{3}-4 a_{2}^{2}\right) z^{2}+\cdots, \quad \frac{w g^{\prime \prime}(w)}{g^{\prime}(w)}=-2 a_{2} w+\left(8 a_{2}^{2}-6 a_{3}\right) w^{2}+\cdots
$$


By using (2.4), (2.5), (2.26), and (2.27), we have

$$
\begin{aligned}
2 a_{2} & =A_{0} B_{1} b_{1}, \\
6 a_{3}-4 a_{2}^{2} & =A_{1} B_{1} b_{1}+A_{0}\left(B_{1} b_{2}+B_{2} b_{1}^{2}\right), \\
-2 a_{2} & =A_{0} B_{1} c_{1}, \\
8 a_{2}^{2}-6 a_{3} & =A_{1} B_{1} c_{1}+A_{0}\left(B_{1} c_{2}+B_{2} c_{1}^{2}\right) .
\end{aligned}
$$

From (2.28) and (2.30), we have

$$
\mathrm{b}_{1}=-\mathrm{c}_{1} .
$$

Squaring and adding (2.28) and (2.30), and then using (2.32), we have

$$
4 \mathrm{a}_{2}^{2}=\mathrm{A}_{0}^{2} \mathrm{~B}_{1}^{2} \mathrm{c}_{1}^{2} \text {. }
$$

Adding (2.29) and (2.31), and then using (2.32), we have

$$
4 \mathrm{a}_{2}^{2}=\mathrm{A}_{0}\left[\mathrm{~B}_{1}\left(\mathrm{~b}_{2}+\mathrm{c}_{2}\right)+2 \mathrm{~B}_{2} \mathrm{c}_{1}^{2}\right] \text {. }
$$

By using (2.2) and (2.3), we have from (2.33) and (2.34) that

$$
\left|\mathrm{a}_{2}\right| \leqslant \frac{\mathrm{B}_{1}}{2} \text { and }\left|\mathrm{a}_{2}\right| \leqslant \frac{\sqrt{\mathrm{B}_{1}+\left|\mathrm{B}_{2}\right|}}{2},
$$

respectively. So we get the desired estimate on the coefficient $\left|a_{2}\right|$ as asserted in (2.25).

Next, in order to find the bound on the coefficient $\left|a_{3}\right|$, we subtract (2.31) from (2.29). Thus, we get

$$
12 a_{3}-12 a_{2}^{2}=A_{1} B_{1}\left(b_{1}-c_{1}\right)+A_{0}\left[B_{1}\left(b_{2}-c_{2}\right)+B_{2}\left(b_{1}^{2}-c_{1}^{2}\right)\right] .
$$

Therefore the equations (2.32), (2.33), and (2.34) yield

$$
12 a_{3}=3 A_{0}^{2} B_{1}^{2} c_{1}^{2}-2 c_{1} A_{1} B_{1}+A_{0} B_{1}\left(b_{2}-c_{2}\right) .
$$

Thus, we find by using (2.2) and (2.3) that

$$
\left|a_{3}\right| \leqslant \frac{1}{4} B_{1}^{2}+\frac{1}{3} B_{1} .
$$

Also from (2.32), (2.34), and (2.35), we have

$$
12 a_{3}=3 A_{0}\left[B_{1}\left(b_{2}+c_{2}\right)+2 B_{2} c_{1}^{2}\right]-2 c_{1} A_{1} B_{1}+A_{0} B_{1}\left(b_{2}-c_{2}\right) .
$$

Hence, we find by (2.2) and (2.3) that

$$
\left|\mathrm{a}_{3}\right| \leqslant \frac{5}{6} \mathrm{~B}_{1}+\frac{1}{2}\left|\mathrm{~B}_{2}\right| .
$$

This evidently completes the proof of Theorem 2.8.

Theorem 2.9. If $\mathrm{f}$ given by (1.1) is in the subclass $\mathrm{C}(\Sigma, \mathrm{h})$, then

$$
\left|a_{2}\right| \leqslant \min \left\{\frac{B_{1}}{2}, \sqrt{\frac{B_{1}+\left|B_{2}\right|}{2}}\right\},
$$

and

$$
\left|a_{3}\right| \leqslant \min \left\{\frac{B_{1}^{2}}{4}+\frac{B_{1}}{6}, \frac{2}{3} B_{1}+\frac{\left|B_{2}\right|}{2}\right\} .
$$

Proof. The result is obvious, by taking $\phi(z) \equiv 1 \quad(z \in \mathcal{U})$ and using the similar procedure as in Theorem 2.8. 


\section{Conclusion}

In the paper, classes of analytic bi-univalent are introduced with the help of quasi-subordination. Further, coefficient estimates of initial Maclaurin coefficients are also obtained.

\section{Acknowledgment}

This project is supported by College of Science Research Center, Deanship of Scientific Research, King Saud University. Author is also thankful to anonymous reviewers for their fruitful comments.

\section{References}

[1] D. A. Brannan, J. Clunie, W. E. Kirwan, Coefficient estimates for a class of star-like functions, Canad. J. Math., 22 (1970), 476-485. 1, 1

[2] D. A. Brannan, T. S. Taha, On some classes of bi-univalent functions, Studia Univ. Babe-Bolyai Math., 31 (1986), 70-77. 1

[3] M. Çağlar, H. Orhan, N. Yağmur, Coefficient bounds for new subclasses of bi-univalent functions, Filomat, 27 (2013), 1165-1171. 1

[4] E. Deniz, Certain subclasses of bi-univalent functions satisfying subordinate conditions, J. Class. Anal., 2 (2013), 49-60. 1

[5] P. L. Duren, Univalent functions, Grundlehren der Mathematischen Wissenschaften [Fundamental Principles of Mathematical Sciences], Springer-Verlag, New York, (1983). 1

[6] S. P. Goyal, P. Goswami, Estimate for initial Maclaurin coefficients of bi-univalent functions for a class defined by fractional derivatives, J. Egyptian Math. Soc., 20 (2012), 179-182. 1

[7] M. Haji Mohd, M. Darus, Fekete-Szeg̋̋ problems for quasi-subordination classes, Abstr. Appl. Anal., 2012 (2012), 14 pages. 1

[8] M. Lewin, On a coefficient problem for bi-univalent functions, Proc. Amer. Math. Soc., 18 (1967), 63-68. 1

[9] Z. Nehari, Conformal Mapping, Reprinting of the 1952 edition, Dover, New York, NY, USA, (1975). 2

[10] E. Netanyahu, The minimal distance of the image boundary from the origin and the second coefficient of a univalent function in $z<1$, Arch. Rational Mech. Anal., 32 (1969), 100-112. 1

[11] F. Y. Ren, S. Owa, S. Fukui, Some inequalities on quasi-subordinate functions, Bull. Austral. Math. Soc., 43 (1991), 317-329. 1

[12] M. S. Robertson, Quasi-subordination and coefficient conjectures, Bull. Amer. Math. Soc., 76 (1970), 1-9. 1

[13] H. M. Srivastava, A. K. Mishra, P. Gochhayat, Certain subclasses of analytic and bi-univalent functions, Appl. Math. Lett., 23 (2010), 1188-1192. 1

[14] Q.-H. Xu, H.-G. Xiao, H. M. Srivastava, A certain general subclass of analytic and bi-univalent functions and associated coefficient estimate problems, Appl. Math. Comput., 218 (2012), 11461-11465. 1 